\title{
Médiévales
}

Langues, Textes, Histoire

73 | automne 2017

Le texte à l'épreuve du numérique

\section{Le texte médiéval à l'épreuve du numérique}

\section{Anne Rochebouet}

\section{OpenEdition}

Journals

Édition électronique

URL : https://journals.openedition.org/medievales/8163

DOI : 10.4000/medievales.8163

ISSN : $1777-5892$

\section{Éditeur}

Presses universitaires de Vincennes

\section{Édition imprimée}

Date de publication : 15 décembre 2017

Pagination : 5-12

ISBN : 978-2-84292-801-8

ISSN : 0751-2708

\section{Référence électronique}

Anne Rochebouet, "Le texte médiéval à l'épreuve du numérique », Médiévales [En ligne], 73 | automne 2017, mis en ligne le 15 décembre 2017, consulté le 22 avril 2022. URL : http://

journals.openedition.org/medievales/8163; DOI : https://doi.org/10.4000/medievales.8163 


\section{Anne Rochebouet}

\section{Le texte médiéval à l'épreuve du numérique}

Depuis les travaux de Roberto Busa qui commença à utiliser, à partir de 1949, le traitement automatique des données pour réaliser entre autres des concordances pour l'œuvre de Thomas d'Aquin ${ }^{1}$, les humanités seraient désormais devenues, du moins en partie, numériques. Si l'omniprésence du syntagme, ou de son équivalent anglo-saxon digital humanities, dans les projets de recherche comme dans le champ institutionnel de l'enseignement supérieur et de la recherche atteste autant d'une réalité des pratiques que d'un effet de mode, voire d'un certain opportunisme dans un contexte où toute la société est présentée comme en pleine et nécessaire mue numérique, il est indéniable que les dix dernières années ont vu fondamentalement changer le mode d'accès et d'exploitation des données de la recherche. Les études médiévales, quelle que soit leur discipline, ne font pas exception ${ }^{2}$ et les projets mettant en jeu des outils numériques se sont multipliés, comme les publications sur le sujet.

Face à ce champ en plein essor, l'objectif de la journée d'études qui a eu lieu en juin 2016 à l'Université de Versailles Saint-Quentin, en partenariat avec l'Université Laval à Québec et avec le labex Patrima, était double. Il

1. <http://www.corpusthomisticum.org/index.html>. C'est généralement ce projet qui est cité comme précurseur des humanités numériques ; voir par exemple L. BURNARD, «Du literary and linguistic computing aux digital humanities : retour sur 40 ans de relations entre sciences humaines et informatique », dans P. MounIER éd., Read/Write Book 2 : une introduction aux humanités numériques, Marseille, OpenEdition Press, 2012, p. 45-58, disponible sur Internet : <http://books.openedition.org/oep/242>, DOI : 10.4000/books. oep.242.

2. On peut même noter que les médiévistes ont souvent très tôt utilisé les possibilités offertes par l'informatique, puis le numérique (on citera par exemple la revue Le Médiéviste et l'ordinateur, créée en 1979 et active jusqu'en 2007 : voir les premiers numéros sur le site Ménestrel, http://www.menestrel.fr/spip.php?rubrique667, puis sur le site de l'IRHT, <http:// lemo.irht.cnrs.fr/>). 
s'agissait d'une part de s'inscrire résolument dans une logique pragmatique et technique, en présentant des dispositifs numériques à partir des contraintes et objectifs ayant guidé leur élaboration ; de l'autre de se situer dans un dialogue entre les disciplines, mais aussi entre chercheurs et professionnels de la conservation et de l'information. En effet, l'omniprésence aujourd'hui des outils et du médium numériques, tout comme les fortes incitations à les utiliser qui pèsent sur les chercheurs, rendent indispensables l'échange et les retours d'expérience sur les dispositifs techniques adoptés ou mis en place par les uns ou les autres, et ce afin de favoriser leur réutilisation, comme celle des données produites, dans le cadre d'autres projets. Cela est d'autant plus nécessaire que les chercheurs, comme leurs équipes, ne disposent pas toujours des moyens techniques, humains et/ou financiers pour élaborer de tels outils ${ }^{3}$.

Pérennité et interopérabilité se veulent donc ici autant techniques (prise en compte et réflexion sur les normes, qui visent à assurer la compatibilité des différents formats et protocoles, mais aussi à garantir l'accès, sur un temps long, aux données produites face à l'évolution des machines et des langages ${ }^{4}$ ) que disciplinaires : il s'agit alors pour chacun, dans la mesure du possible, de penser l'exploitation numérique de son objet d'étude de manière à ce qu'il puisse également être utilisé dans le cadre de recherches menées avec des méthodes et des objectifs différents. Les professionnels de la conservation et de l'information qui travaillent dans le monde des archives, des musées et des bibliothèques ont également développé une grande expertise dans ces domaines, qui touchent directement un certain nombre de leurs missions (accès aux collections et aux fonds, valorisation par le développement d'outils de signalement et de recherche, conservation, notamment par la création de supports de substitution). Ils sont ainsi partie prenante de nombreux projets et groupes de réflexion (en particulier sur la constitution de normes internationales), dans une perspective de mise à disposition des collections et d'archivage des données qui n'est pas celle des chercheurs, mais dont ces derniers bénéficient.

C'est ainsi en s'appuyant avant tout sur des études de cas variées, par les corpus étudiés (du point du vue chronologique, géographique, linguistique comme quantitatif), par les méthodes et champs disciplinaires sollicités (histoire, philologie, linguistique, paléographie, littérature, etc.) et enfin par les moyens humains, techniques et financiers mis en œuvre,

3. S'ajoute également la question de la pérennité des moyens alloués au projet, le numérique pouvant générer des coûts d'hébergement et nécessitant surtout un certain nombre d'opérations de maintenance et de mise à jour, une fois le projet en lui-même terminé.

4. Voir par exemple le travail des grands consortiums internationaux comme le World Wide Web Consortium (W3C), <https://www.w3.org/>, ou, concernant plus précisément les formats d'encodage et d'archivage des textes numériques, celui de la TEI (Text Encoding Initiative), <http://www.tei-c.org>. 
que ce dossier voudrait croiser les différentes utilisations, pour l'étude et l'exploitation des textes médiévaux, du support numérique comme des outils développés grâce au traitement automatique des données.

Les huit contributions qui vont suivre ont également comme point commun d'être centrées sur l'analyse de données textuelles. Cette limitation au texte, soit à toute " chaîne linguistique [ici] écrite formant une unité communicationnelle ${ }^{5}$ », explique l'absence de projets relevant du traitement du matériau linguistique pour lui-même (par exemple ceux menés dans le cadre de recherches lexicographiques au sens strict) : c'est ici l'unité textuelle et les analyses qui portent sur cette dernière qui sont mises en jeu, et non l'exploitation de segments linguistiques, utilisés indépendamment de l'ensemble dans lequel ils ont été puisés.

Le choix de se limiter à l'exploitation des textes n'implique pas, en revanche, que seul le contenu sémantique transmis par ce dernier soit celui envisagé ; la forme et l'inscription du texte sur son support font également partie, même si c'est souvent de manière secondaire comme nous le verrons, d'une partie des dispositifs présentés, et au-delà de bon nombre de projets en cours. Ce lien entre le texte et sa matérialité pourrait paraître paradoxal, la numérisation étant par essence une dématérialisation. Force est de constater, cependant, que la plupart des projets de recherche impliquant un recours au numérique associent textes et images, contenu sémantique et visualisation, ou prise en compte du ou des support(s) qui le conserve(nt) et qui l'informe(nt) : il est par exemple rare aujourd'hui de trouver une édition électronique de texte qui ne comporterait pas de renvois aux reproductions numérisées du ou des manuscrit(s) ou document(s) utilisé(s) ; de même, dans le langage XML-TEI ${ }^{6}$, l'un des plus utilisés actuellement par la communauté scientifique pour les éditions et publications en ligne, les balises servant à décrire la mise en page et la forme du support sont parmi les plus développées ${ }^{7}$. La présence de l'image ou de la mémoire de la forme originelle du texte doit cependant être diversement interprétée ${ }^{8}$ : elle est notamment en partie significative de la façon dont le support numérique

5. Le texte peut bien sûr connaître des manifestations écrites ou orales, comme le précisait la formulation complète de cette définition tirée de l'article « Texte » du Nouveau Dictionnaire encyclopédique des sciences du langage (O. DUCROT et J-M. SCHAEFFER éd., Paris, 1995, p. 594-607, ici p. 594), mais c'est ici à sa forme écrite, pour des raisons évidentes, que nous nous limiterons.

6. La TEI (Text Encoding Initiative, voir note 4) utilise désormais le langage XML (Extensible Markup Language).

7. Et non celles permettant de décrire, par exemple, les variations que peut connaître un texte, qu'on envisage ces dernières comme des variantes face à un archétype, un ou des avant-texte(s) vs un texte, etc.

8. En premier lieu, la présence de l'image y résulte en effet moins, le plus souvent, d'un postulat méthodologique (le texte n'est pleinement actualisé que par le support physique et/ ou matériel qui l'informe) que de la prise en compte des attendus visuels et ergonomiques 
brouille, pour le lecteur qu'est le chercheur, les différents statuts du texte.En cela, ce support met à l'épreuve notre notion du texte comme les questions que nous lui posons, tout en étant un formidable révélateur de nos choix méthodologiques comme de nos paradigmes épistémologiques ; et c'est sur cette labilité, exposée et renforcée par le numérique, que je voudrais m'arrêter ici.

Il n'est pas inutile de rappeler que l'emploi que nous faisons du terme «numérique » le place à la fois du côté de l'outil (il renvoie alors au traitement automatique, par la machine, de données ${ }^{9}$ ) et du côté du médium (moyen d'accès et, via l'écran, d'appréhension et de mise en forme des données). Le Digital Turn ou la « conversion numérique ${ }^{10}$ » implique ainsi toujours une migration des données qui, avec le développement de l'Internet, s'accompagne très souvent d'une publication, c'est-à-dire de leur mise à disposition, en accès libre ou restreint, sur le Web. Le travail de publication et d'édition en ligne qui en résulte consiste ainsi à traduire le texte, augmenté ou non d'un autre type de contenu, en du code informatique « destiné à être interprété par des systèmes techniques sur lesquels l'éditeur n'a pas de maîtrise ${ }^{11} »$; parmi ce code informatique doivent également figurer des métadonnées, qui identifient et décrivent, pour la machine, ce qui a été codé. Le producteur de texte ainsi numérisé, que ce dernier soit ensuite destiné à une lecture linéaire ou à une consultation par l'intermédiaire d'une base de données, est donc aussi et avant tout un méta-éditeur, c'est-à-dire qu'il ne fixe plus la forme du texte ${ }^{12}$ qu'il publie ou établit, mais lui donne une place dans un système de référencement en programmant les outils informatiques qui lui donneront forme à sa place. Cela est d'autant plus vrai à l'heure du Web sémantique, parfois appelé Web 3.0, où le lien est fait directement entre les données, et non plus entre les pages.

Dans le cas des textes médiévaux, les données de départ qui sont ainsi converties ont des statuts très différents, qu'uniformisent cependant, une fois sur l'écran, à la fois le manque de métadonnées (ou l'inadéquation de certains champs communément utilisés) et la mise en forme, souvent standardisée, appliquée par les logiciels, et notamment les logiciels de

de l'utilisateur du web 3.0, habitué aux images comme aux liens vers des reproductions numériques des documents sollicités.

9. Par exemple la textométrie ou le TAL (traitement automatique des langues), la linguistique de corpus, les statistiques, les bases de données, etc. Il s'agit notamment d'exploiter les fameuses données massives, ou big data.

10. M. DoueIHI, La Grande Conversion numérique, Paris, 2008 pour la traduction française.

11. P. Mounier, «Le Livre et les trois dimensions du cyberespace », dans M. DACOs éd., Read/Write Book : le livre inscriptible, Marseille, 2010, p. 175-183, disponible sur Internet : $<$ http://books .openedition.org/oep/179>, DOI : 10.4000/books.oep.179.

12. Forme du texte dont les épreuves, sur le support papier, contrôlaient justement l'adéquation au projet de départ. 
gestion de contenu ${ }^{13}$. Une édition critique de la seconde moitié du XIX ${ }^{\mathrm{e}}$ siècle, tombée dans le domaine public et accessible via de grandes plateformes de numérisation comme archive.org, n'obéit pas aux mêmes objectifs ni à la même méthodologie que la transcription directement effectuée sur un seul témoin dans le cadre d'un projet de recherche (ou d'une opération de crowdsourcing ${ }^{14}$ ), ou encore que l'océrisation ${ }^{15}$ d'une édition imprimée du $\mathrm{XVI}^{\mathrm{e}}$ ou du XVIII ${ }^{\mathrm{e}}$ siècle, numérisée en mode image et disponible sur Gallica. Le numérique brouille ainsi, bien davantage que la publication papier, la distinction entre transcription de travail, fac-similé et édition critique - quels que soient les critères méthodologiques qui guident cette dernière.

Mais cette labilité de statut du texte numérique ne relève pas d'une simple problématique de critique des sources, bien connue par ailleurs des médiévistes ${ }^{16}$. L'inventaire rapide, dressé plus haut, des différentes

13. En anglais CMS (Content Management System). Voir par exemple A. SAEMMER, «Lire la littérature numérique à l'université : deux situations pédagogiques », Ela. Études de linguistique appliquée, 160 (2010/4), p. 411-420, disponible en ligne : <http://www.cairn. info/revue-ela-2010-4-page-411.htm>.

14. Le crowdsourcing, ou production participative, est une pratique à l'origine très développée dans le monde des bibliothèques anglo-saxonnes ; elle s'est récemment multipliée, notamment dans les projets numériques patrimoniaux. Elle consiste à solliciter des internautes pour participer bénévolement aux travaux souvent très longs de transcription, de référencement ou encore de balisage de documents. Voir par exemple, dans les projets récents, le « transcribathon » organisé par la bibliothèque numérique Europeana (http://www. europeana.eu/portal/fr) autour de documents de la période 1914-18 (<https://transcribathon. com/en/>). Les projets concernant l'époque médiévale sont plus rares, mais existent, par exemple en Espagne, Deciphering Secrets, Treasures of the Archive of the Cathedral of Burgos (<http://www.decipheringsecrets.com/>) ou, aux États-Unis, The Medieval Fragments Project (M. ERWIN, « Fragments of Medieval Manuscripts in Printed Books : Crowdsourcing and Cataloging Medieval Manuscript Waste in the Book Collection of the Harry Ransom Center », Manuscripta, 60-2 (2016), p. 188-247, disponible en ligne : <https:// doi.org/10.1484/J.MSS.5.111918>).

15. Reconnaissance Optique de Caractères, en anglais OCR.

16. Au-delà du cercle des utilisateurs et lecteurs spécialisés que sont les médiévistes, on notera que cette question de l'évaluation du statut du texte de départ est loin d'être évidente pour bon nombre d'internautes. On s'arrêtera à titre d'exemple sur les échanges sur le Web auxquels a pu donner lieu la procédure juridique auprès du tribunal de grande instance de Paris engagée entre deux maisons d'édition de textes critiques médiévaux et jugée en mars 2014 (voir par exemple le billet « Une victoire pour le domaine public : un cas de copyfraud reconnu par un juge français » publié le 13 avril 2014 sur le blog S.I.Lex par Lionel Maurel, bibliothécaire <https://scinfolex.com/2014/04/13/une-victoire-pour-le-domaine-public-uncas-de-copyfraud-reconnu-par-un-juge-francais/>, ou celui du 14 avril 2014 par Maïeul Rouquette, docteur en théologie et en histoire de l'Université de Lausanne, " Du droit d'auteur appliqué aux éditions critiques » sur son carnet de recherche Apocryphes, <http:// apocryphes.hypotheses.org/389>). La procédure visait des textes médiévaux français dont l'édition ou une partie de l'édition, dépourvue de son apparat, avait été réutilisée dans une base de données mise au point par une autre maison d'édition que celle dans laquelle le texte avait été publié à l'origine en format papier. Une partie des échanges sur le Web se sont centrés sur la notion de copyfraud (c'est-à-dire sur l'extension abusive d'un droit de propriété 
opérations que nécessite l'utilisation de données textuelles numériques (et qui conditionnent en partie l'exploitation qui peut en être faite) permet de voir que la difficulté est renforcée par les spécificités du médium numérique. Notre appréhension des textes y est en effet guidée par une double illusion mimétique, celle de l'écran et celle de la machine. Comme le soulignent nombre de travaux des sciences de l'information et de la communication, l'écran, notamment par l'usage extensif qu'il fait désormais de l'image ${ }^{17}$, est considéré implicitement comme garantissant un accès direct, non médié, entre l'utilisateur et les données, par exemple ici le manuscrit ; la machine quant à elle est perçue comme gage d'une «objectivité computationnelle ${ }^{18}$ » : instrument mécanique, elle apparaît comme un moyen d'appréhension neutre et objectif de ces mêmes données. Or toute migration de support implique une opération de transposition, qui ne peut être blanche, mais s'appuie au contraire sur une série de choix, et donc d'interprétations.

À ces illusions mimétiques, largement impensées, s'ajoute le poids des pratiques, qu'il ne faut pas négliger, car elles encouragent des usages en créant des horizons d'attente formels comme intellectuels. C'est ainsi d'abord comme formidable démultiplicateur d'accès libre et ouvert aux collections, aux fonds, et donc aux documents et aux objets qu'a été et qu'est toujours utilisé le numérique par les institutions de conservation : elles ont ainsi particulièrement développé, dans la dernière décennie notamment ${ }^{19}$, la numérisation de leurs collections (entendue ici comme la migration vers un support numérique permettant une visualisation en mode image, accompagnée d'un référencement et/ou d'un balisage plus ou moins fin). C'est en partie dans la suite de ce mouvement que les projets utilisant le numérique intègrent très souvent dans leurs objectifs premiers de donner accès aux documents « primaires » sur lesquels sont basées leurs recherches. Cette problématique d'accès aux documents et aux sources

intellectuelle sur des données considérées comme appartenant au domaine public, et devant de ce fait être en libre accès) et donc, en filigrane, sur la notion de texte qui détermine en partie l'existence d'un tel droit de propriété. Il ne s'agit pas ici de prendre position sur la légitimité de la revendication juridique de l'une ou l'autre partie, ni d'ailleurs sur celle du libre accès des données produites par la recherche, mais bien de souligner le brouillage qui s'est opéré, dans bon nombre de billets d'internautes sur ce sujet, entre l'objet manuscrit sous sa forme numérisée, considéré comme relevant du domaine public, le texte qu'il contient (qui peut n'être que l'une des versions de l'œuvre), et l'hypothèse de travail, aussi informée et soignée soit-elle, qu'est le texte établi d'après un ou plusieurs témoins par l'éditeur critique, ou par le simple transcripteur.

17. Cet usage extensif est rendu possible par les évolutions techniques, qui permettent désormais de gérer les masses de données générées par les images, comme par la multiplication des numérisations en mode image accessibles en libre accès.

18. J'emprunte ce concept aux travaux de L. J. DASTON et P. GALISON sur l'histoire du concept d'objectivité scientifique (Objectivity, Cambridge (MA), 2007).

19. Le site de Gallica, projet pionnier de la Bibliothèque nationale de France de bibliothèque numérique, a été ouvert sous sa première forme en 1997. 
entraîne un recours massif, mais finalement peu interrogé, à des types de traitement et de présentation perçus comme descriptifs ou documentaires, et à une appréhension du numérique et de l'Internet comme lieu de dépôt de données qui seraient, comme on l'a vu, non médiées. C'est ainsi une sorte de culture, visuelle et intellectuelle, du fac-similé qui est pour l'instant prégnante dans les projets numériques, aux dépens de la réflexion sur les différents statuts du texte par rapport au document ou l'objet qui le contient. En s'appuyant ainsi sur l'idée que le passage au numérique est une opération neutre, favorisée par une forme de mimétisme technique (la reprise sans l'interroger du protocole technique proposé par un autre projet), c'est alors l'importance des choix effectués par tout transcripteur ou éditeur qu'on risque de gommer, comme la différence entre le texte, l'œuvre et le (ou les) document(s).

C'est entre autres ce que souligne Frédéric Duval dans sa synthèse sur les pratiques actuelles de l'édition de textes numérique, qui ouvre le dossier. Dans le cas des éditions de textes médiévaux français, auxquels il se limite ici, il montre ainsi que c'est l'édition de type documentaire, celle qui veut donner accès à un témoin (document) et non au texte ou à l'œuvre (work), qui est pour l'instant largement répandue.

Les sept contributions qui suivent présentent des retours d'expérience sur des projets en cours ainsi que sur les outils informatiques développés ou adoptés dans leur cadre, comme sur les perspectives offertes par ces derniers. Du côté des outils conçus à partir d'éditions de texte nativement numériques ou de textes numérisés, c'est d'abord la gestion des données prosopographiques qui retient Graziella Pastore : à partir d'un corpus restreint, tiré de l'édition électronique d'une compilation juridique en français médiéval, le Livre de jostice et de plet, elle détaille comment constituer une base de données avec le logiciel libre Omeka. C'est également sur les entités nommées (personnages, institutions, lieux) que porte le travail de Sergio Torres Aguilar, qui, par l'automatisation de leur identification à l'intérieur des chartes latines préalablement numérisées au sein du Corpus Burgundiae Medii Aevi, vise à créer un outil d'interrogation, et au-delà de structuration de telles bases, tout en prenant en compte la spécificité des documents médiévaux et des questionnements qui leur sont appliqués. La confrontation de la machine aux manuscrits médiévaux est de même au centre du projet HIMANIS (HIstorical MANuscript Indexing for user-controlled Search), présenté ici par Dominique Stutzmann, Sébastien Hamel et Jean-François Moufflet ; 1'objectif en est d'indexer les registres de la chancellerie royale française (1302-1483) conservés aux Archives nationales à partir des images numérisées des manuscrits, mais aussi des index et éditions imprimées existantes. Il s'agit autant de réfléchir à la manière pour la machine d' " apprendre » à déchiffrer des manuscrits qu'à la façon de donner accès aux données produites en fonction d'un modèle 
interprétatif pertinent. La gestion des données massives, les big data, sont ainsi la toile de fond de ces différents projets.

Les contributions de Xavier-Laurent Salvador et Thibault Clérice présentent quant à elles deux types différents de gestionnaires de contenu numérique, en s'interrogeant notamment sur la modélisation et la structuration, permises par ce biais, des données textuelles. À partir de l'exemple de la Bible historiale de Guyart des Moulins, première traduction intégrale commentée en prose française médiévale, Xavier-Laurent Salvador décrit le principe de fonctionnement d'Isilex, outil de saisie et de mise en ligne de corpus automatisés couplant XML et logique REST XQuery. Thibault Clérice expose de son côté les différentes fonctionnalités de CapiTainS, outil qui fournit un ensemble de services et d'interfaces pour l'exploitation des publications numériques et de leurs données.

Les projets présentés peuvent enfin entrer dans la logique cumulative et collaborative de la plate-forme ou du portail dont l'objectif est de structurer des informations de statut et de types variés, réunies petit à petit, pour mieux appréhender les corpus d'études ainsi constitués. Anne Salamon fait la genèse de la mise au point du portail $\mathrm{H}$ (istoires) $\mathrm{U}$ (niverselles) 15 , qui doit permettre d'étudier les histoires universelles du $\mathrm{XV}^{\mathrm{e}}$ siècle, corpus historique de langue française particulièrement difficile d'accès du fait de sa taille mais aussi du caractère inédit et mal connu de la plupart des textes concernés. Davide Gherdevich expose de son côté le fonctionnement du site collaboratif POLIMA (POuvoirs de la LIste au Moyen Âge), basé sur MediaWiki et Semantic MediaWiki, qui vise à la fois à créer un corpus de travail et un moyen de questionner, dans une perspective interdisciplinaire, ce dernier.

Tous ces projets interrogent les possibilités et les contraintes générées par le traitement numérique des données textuelles médiévales. Au-delà de l'intérêt spécifique de chaque étude de cas, leur réunion dans ce dossier thématique a enfin pour objectif de dépasser la simple revue des pratiques pour appréhender ce que le numérique fait au texte médiéval, ou, pour le dire autrement, comment les usages numériques actuels guident et influencent notre appréhension de ces textes comme de leurs supports, et informent donc en partie les questions que nous leur posons par le biais de ces dispositifs numériques.

Anne Rochebouet - DYPAC, Université de Versailles Saint-Quentin (ParisSaclay) 